\title{
EFICACIA DE LA TÉCNICA DE TENSIÓN APLICADA PARA EL CONTROL DEL SÍNDROME VASOVAGAL APLICADA A UN CASO DE HEMATOFOBIA
}

\author{
MERCEDES BORDA, OLVIDO MARTÍNEZ Y ALFONSO BLANCO \\ Universidad de Sevilla
}

(Recibido el 10 de noviembre de 1997)

\begin{abstract}
En el presente trabajo se describe el tratamiento y evolución de una persona con fobia a la sangre con un seguimiento de 12 meses. Se utilizó una combinación de dos técnicas: "exposición gradual en vivo" a las situaciones fóbicas y la "tensión aplicada" para aprender a controlar el síndrome vasovagal y prevenir el desmayo. La evaluación se realizó en el pretratamiento, postratamiento y en los seguimientos de 1, 6 y 12 meses. El tratamiento constó de 6 sesiones en un período de 6 semanas, con una duración aproximada $90 \mathrm{mn}$. por sesión. En los resultados se observa una mejoría significativa a corto y largo plazo en todas las medidas realizadas.
\end{abstract}

Palabras clave: Hematofobia, exposición en vivo, tensión aplicada, eficacia terapéutica.

\section{Applied Tension technique's efficacy for the vasovagal syncope applied to a blood phobic patient}

In this paper the treatment and evolution of a blood-phobia patient is described, with a follow-up of twelve months. A combination of two techniques was used: "gradual exposure in vivo" to phobic situations and the "applied tension" to learn to control the vasovagal syncope and to prevent fainting. The evaluation was made in the pretreatment, post-treatment and along the follow-ups of one, six and twelve months. The treatment consisted of six sessions in a period of six weeks, with an approximate duration of ninety minutes per session. On the results, a significant improvement in short and long term is observed in all the measurements taken.

Key words: Blood-phobia, exposure in vivo, applied tension, therapeutic efficacy.

\section{INTRODUCCIÓN}

La fobia a la sangre, definida como el miedo y la evitación de situaciones relacionadas directa $\mathrm{o}$ indirectamente con la sangre, las inyecciones y las heridas (Marks, 1988) es la fobia específica más común en la población general. Se observa con mayor frecuencia en mujeres (Öst,

Correspondencia: Departamento de Psiquiatría, Personalidad, Evaluación y Tratamiento Psicológicos, Facultad de Psicología, Universidad de Sevilla.

Avenida San Francisco Javier, s/n, 41005 SEVILLA Teléf: 954557806 . Fax: 954557807.

Este trabajo procede de la actividad del Grupo de Investigación y Desarrollo Tecnológico $n^{*} 1012$ de la Junta de Andalucia denominado Psicología Clínica y de la Salud, dirigido por D. Alfonso Blanco Picabia.
Sterner y Lindahl, 1984) y suele comenzar en la infancia, dándose un elevado porcentaje de casos con antecedentes familiares (Öst, 1992).

Este miedo comparte con las otras fobias la adopción de conductas de evitación y/o escape de las situaciones fóbicas (por ejemplo, extracciones de sangre o visión de películas violentas) y la presencia de pensamientos anticipatorios en relación con dichas situaciones, bien por recordar lo sucedido en situaciones anteriores con una gran carga de ansiedad, bien por el conocimiento de experiencias negativas acaecidas a alguna persona cercana (por ejemplo, "voy a caerme delante de todos", "no voy a ser capaz de 
aguantar sin desmayarme»). Sin embargo, en cuanto a las respuestas psicofisiológicas, los fóbicos a la sangre presentan un patrón de respuesta específica o "respuesta bifásica" (Page, 1994). En la primera fase, junto con otros síntomas de ansiedad, se da un aumento principalmente del ritmo cardíaco (taquicardia), de la presión arterial y de la tasa respiratoria. En la segunda fase, estos parámetros descienden bruscamente, siendo tal el enlentecimiento del ritmo cardíaco (bradicardia de hasta 30-45 pulsaciones por minuto) y la disminución de la presión sanguínea que en casos extremos puede llevar al desmayo (Vázquez y Buceta, 1990).

Otro aspecto que también marca su diferencia de otras fobias, es el hecho de que la respuesta de evitación se produce más por el temor al desmayo que por el miedo a las situaciones fóbicas propiamente dicho (Marks,1987).

Las situaciones más evitadas suelen ser las relacionadas con las extracciones de sangre, pero este fenómeno también puede llegar a interferir con procesos médicos de mayor importancia (por ejemplo, rechazar intervenciones quirúrgicas) o en áreas tales como la elección de los estudios (por ejemplo, rechazar Enfermería o Medicina) o en la toma de decisiones tan vitales como en una mujer no querer quedarse embarazada por temor al parto (Borda, Antequera y Blanco Picabia, 1996).

A pesar de su alta prevalencia en la población adulta $(3,1 \%$, Agras, Silvester y Oliveau, 1969) es escasa la demanda de ayuda terapéutica, si bien esto puede explicarse en que no se muestra como una fobia de excesiva incapacitación debido a la instauración de los estilos de conducta. Añadir que este tipo de fobia no remite espontáneamente y suele persistir en el tiempo.

El objetivo de este trabajo es mostrar la eficacia de la técnica de «tensión aplicada»; es decir, de la exposición gradual en vivo combinada con la técnica de «tensión muscular», aplicada en un varón joven afectado por la hematofobia.

\section{MÉTODO}

\section{Sujeto}

Nuestro paciente es un adolescente de 15 años de edad, estudiante de BUP. Es el hermano mediano de una familia formada por tres hijos, 2 varones y una niña menor, de 8 años. Acude a consulta en respuesta a la divulgación en la prensa sobre la puesta en práctica de un programa de tratamiento psicológico para personas afectadas por la hematofobia.

Afirma tener el problema «desde siempre» refiriéndonos que el primer episodio que recuerda fue en el colegio. El suceso tuvo lugar durante la visualización de un vídeo sobre un parto, momento en el cual se mareó. También nos dice que, desde que es capaz de recordar, siempre se ha mareado al hacerse un análisis de sangre.

Desde aquel primer episodio, el problema ha evolucionado hasta el punto de evitar cualquier situación relacionada con la sangre, tal como hacerse una analítica o entrar en clínicas o centros hospitalarios, y en el caso de situaciones desprevenidas, como hacerse una herida (por pequeña que fuera) tiene que ser atendido por otra persona, ya que responde mareándose. Tampoco puede escuchar ninguna conversación, ver en $\mathrm{TV}$, ni leer noticia alguna relacionada con la sangre o las heridas, ni acudir al médico ni al dentista.

Él realmente no sabe describir qué le sucede, sólo sabe que, inmediatamente después de escuchar o ver algo, siente un sudor frío y que las piernas no le responden, se queda quieto, callado, esperando, y sin poder hacer nada por remediarlo, se desvanece. 
Al intentar especificar con más detalle las diferentes respuestas o síntomas, observamos:

a) Que con respecto a las respuestas motoras, lo más característico aparte de la evitación es un excesivo movimiento de las extremidades con temblor de manos y pies.

b) Con respecto a las respuestas psicofisiológicas, destacan como más frecuentes: molestias en el estómago, taquicardia, sudor de manos y axilas, aumento de la respiración, mayor bombeo de sangre con sudor frío, palidez facial y en casos más extremos, el desmayo.

c) En relación con los pensamientos que acompañan antes y durante la situación fóbica, estos hacían referencia a la sensación de «no ser capaz de controlar la situación», de «no aguantar mucho sin marearse», de "no poder reaccionar», y sobre todo, el recuerdo de situaciones negativas pasadas y la anticipación del desmayo.

Derivado de su problema es el hecho de que el paciente no percibe tener casi ningún control de sus reacciones en estas circunstancias por lo que no quiere ni hablar del tema ya que está pensando que en cualquier momento se desmayará.

El paciente se considera una persona sana. No ha recibido anteriormente ningún tipo de tratamiento médico o psicológico por este problema. En su familia hay antecedentes muy cercanos con fobia a la sangre, su madre y su hermano (ambos más leves que él). Aparte de la hematofobia que es el motivo que le trae a consulta, no ha padecido ninguna enfermedad física ni ningún trastorno psicológico. Se ve a sí mismo como una persona tímida, sin un excesivo círculo de amistades pero buenos amigos, interesado en sus estudios, obteniendo muy buenos resultados académicos. No consume alcohol ni tabaco; leer y escuchar música son algunas de sus preferencias.
Es conveniente resaltar que nuestro paciente formó parte de un programa de tratamiento llevado a cabo en varios grupos de 3-4 personas con el tipo de problema y de características semejantes, siendo él miembro de uno de los grupos (nivel de severidad alto), con fines de investigación (Borda, Rodríguez Franco, Antequera y Blanco Picabia, 1998, en prensa).

\section{Profesionales}

El equipo estaba formado por 8 psicólogos, 4 terapeutas y 4 coterapeutas, 1 psiquiatra y un ATS. Los terapeutas tenían al menos 2 años de experiencia clínica en terapia de conducta y los coterapeutas entre 6 y 12 meses. Las sesiones estaban diseñadas previamente por el psicólogo responsable, de tal manera que el desarrollo de cada sesión era guiado por los terapeutas, quienes contaban con la ayuda del coterapeuta.

\section{PROCEDIMIENTO}

\section{Evaluación}

El proceso de evaluación se llevó a cabo en cinco momentos distintos: pretratamiento, postratamiento, seguimientos de 1, 6 y 12 meses.

En el pretratamiento se le pasó al paciente una entrevista clínica, el Inventario de Fobia a la Sangre (Borda, Antequera y Blanco Picabia, 1994), el Cuestionario de Miedos (Marks y Mathews, 1979), la Escala de Conductas Objetivo (Borda, Antequera y Blanco Picabia, 1994), y el Cuestionario de 16 factores de Personalidad: 16 PF (Cattell, 1972). Asimismo, en el postratamiento y los distintos seguimientos se pasaron las tres medidas de evaluación clínica. 
a) Entrevista para la hematofobia (Borda, Antequera y Blanco, 1994)

Es una entrevista semiestructurada para recoger datos personales del paciente y de la historia y evolución del problema hasta el estado actual. También recoge datos como los antecedentes familiares o el grado de afectación del problema a su vida cotidiana, así como sus expectativas del tratamiento. Estå elaborada para ser autoaplicada.

\section{b) Inventario de Fobia a la Sangre (Borda, Antequera y Blanco, 1994)}

Es un inventario específicamente creado para evaluar hematofobia. Está compuesto por 50 items referidos a diversas situaciones relacionadas preferentemente con la sangre, las inyecciones y el dentista, y en menor medida con la sangre animal, el color rojo, la agorafobia y la ansiedad social. Mide en una escala de 0 (nunca) a 3 (siempre) los diferentes tipos de respuestas (motoras, psicofisiológicas y cognitivas) del paciente y valora tanto las respuestas de "ansiedad situacional» como las de «ansiedad anticipatoria» (Tabla 1).

\section{c) Cuestionario de Miedos} (Marks y Mathews, 1979)

Este cuestionario está compuesta por 15 items subdivididos en 3 subescalas de 5 items cada una que miden respectiva-

Tabla 1. Puntuaciones en el Inventario de Fobia a la Sangre en las distintas evaluaciones

\begin{tabular}{|c|c|c|c|c|c|}
\hline Inventario de Hematofobia & Pretrat. & Postrat. & 1 mes & 6 meses & 12 meses \\
\hline $\begin{array}{l}\text { Escala general } \\
\text { Psicofisiológica } \\
\text { Cognitivas } \\
\text { Motoras }\end{array}$ & $\begin{array}{l}651 \\
453 \\
223\end{array}$ & $\begin{array}{r}70 \\
13 \\
7\end{array}$ & $\begin{array}{l}35 \\
19 \\
11\end{array}$ & $\begin{array}{l}35 \\
21 \\
16\end{array}$ & $\begin{array}{r}12 \\
1 \\
2\end{array}$ \\
\hline $\begin{array}{l}\text { Anticipatorias } \\
\text { Psicofisiologicas } \\
\text { Cognitivas } \\
\text { Motoras }\end{array}$ & $\begin{array}{r}205 \\
162 \\
73\end{array}$ & $\begin{array}{r}20 \\
5 \\
2\end{array}$ & $\begin{array}{r}14 \\
8 \\
4\end{array}$ & $\begin{array}{r}9 \\
11 \\
4\end{array}$ & $\begin{array}{l}8 \\
0 \\
0\end{array}$ \\
\hline $\begin{array}{l}\text { Situacionales } \\
\text { Psicofisiológicas } \\
\text { Cognitivas } \\
\text { Motoras }\end{array}$ & $\begin{array}{l}446 \\
291 \\
150\end{array}$ & $\begin{array}{r}50 \\
8 \\
5\end{array}$ & $\begin{array}{r}21 \\
11 \\
7\end{array}$ & $\begin{array}{l}26 \\
10 \\
12\end{array}$ & $\begin{array}{l}4 \\
1 \\
2\end{array}$ \\
\hline $\begin{array}{l}\text { Sub. de Sangre Animal } \\
\text { Psicofisiológicas } \\
\text { Cognitivas } \\
\text { Motoras }\end{array}$ & $\begin{array}{l}1 \\
0 \\
0\end{array}$ & $\begin{array}{l}0 \\
0 \\
0\end{array}$ & $\begin{array}{l}0 \\
0 \\
0\end{array}$ & $\begin{array}{l}0 \\
0 \\
0\end{array}$ & $\begin{array}{l}0 \\
0 \\
0\end{array}$ \\
\hline $\begin{array}{l}\text { Sub. Color Rojo } \\
\text { Psicofisiológicas } \\
\text { Cognitivas } \\
\text { Motoras }\end{array}$ & $\begin{array}{l}0 \\
0 \\
0\end{array}$ & $\begin{array}{l}0 \\
0 \\
0\end{array}$ & $\begin{array}{l}0 \\
0 \\
0\end{array}$ & $\begin{array}{l}0 \\
0 \\
0\end{array}$ & $\begin{array}{l}0 \\
0 \\
0\end{array}$ \\
\hline $\begin{array}{l}\text { Sub. Agorafobia } \\
\text { Psicofisiológicas } \\
\text { Cognitivas } \\
\text { Motoras }\end{array}$ & $\begin{array}{l}21 \\
15 \\
10\end{array}$ & $\begin{array}{l}0 \\
0 \\
0\end{array}$ & $\begin{array}{l}0 \\
0 \\
0\end{array}$ & $\begin{array}{l}0 \\
0 \\
0\end{array}$ & $\begin{array}{l}0 \\
0 \\
0\end{array}$ \\
\hline $\begin{array}{l}\text { Sub. Ansiedad Social } \\
\text { Psicofisiologicas } \\
\text { Cognitivas } \\
\text { Motoras }\end{array}$ & $\begin{array}{r}14 \\
0 \\
0\end{array}$ & $\begin{array}{l}0 \\
0 \\
0\end{array}$ & $\begin{array}{l}0 \\
0 \\
0\end{array}$ & $\begin{array}{l}0 \\
0 \\
0\end{array}$ & $\begin{array}{l}0 \\
0 \\
0\end{array}$ \\
\hline
\end{tabular}


mente hematofobia, agorafobia y fobia social. El paciente debe valorar en una escala de 0 (nunca) a 8 (siempre) puntos la frecuencia de evitación de las situaciones allí señaladas (Tabla 2).

\section{d) Escala de conductas-objetivo}

(Borda, Antequera y Blanco, 1994)

Esta escala consta de 5 items o conductas-problemas que define el propio paciente y son situaciones relacionadas con la sangre que le suponen algún tipo de dificultad y sobre las que se trabajará durante el tratamiento. A la vez de definir las conductas, el paciente debe valorar en 3 subescalas tanto el grado de dificultad actual [medido de 0 (nada) a 10 (máximo) puntos] como la frecuencia de los episodios de desmayo actuales [medido de 0 (nada) a 5 (siempre) puntos] así como la frecuencia de evitación [medida de 0 (nunca) a 5 (siempre) puntos] (Tabla 3).

\section{e) Cuestionario de 16 factores de Personalidad: 16 PF (Cattell, 1972)}

El cuestionario de personalidad de 16 Factores es un instrumento de valoración objetiva, cuyo fin es ofrecer, en el menor tiempo posible, una visión muy completa de la personalidad. Esta visión se basa en la evaluación de 16 dimensiones de personalidad, fundamentalmente independientes y psicológicamente significativas, lo cual nos da mayor posibilidad de comprensión y predicción del comportamiento humano. Consta de 187 items con 3 posibilidades de respuesta (Tabla 4).

Con respecto a los resultados del Cuestionario de Personalidad 16 PF cabe decir que todo el grupo, incluido nuestro

Tabla 2. Puntuaciones obtenidas en el Cuestionario de Miedos en las distintas evaluaciones

\begin{tabular}{|c|c|c|c|c|c|}
\hline Cuestionario de Miedos & Pretrat. & Postrat. & $\begin{array}{c}\text { Seg. } \\
1 \text { mes }\end{array}$ & $\begin{array}{l}\text { Seg. } \\
6 \text { meses }\end{array}$ & $\begin{array}{c}\text { Seg. } \\
12 \text { meses }\end{array}$ \\
\hline Puntuación total & 38 & 13 & 8 & 2 & 0 \\
\hline S. Hematofobia (rango 0-40) & 28 & 7 & 6 & 1 & 0 \\
\hline S. Agorafobia (rango 0-40) & 4 & 4 & $\mathbf{0}$ & 0 & 0 \\
\hline S. Fobia Social (rango 0-40) & 6 & 2 & 2 & 1 & 0 \\
\hline
\end{tabular}

Tabla 3. Puntuaciones en la escala Conductas-Objetivo en las distintas evaluaciones

\begin{tabular}{|c|c|c|c|c|c|}
\hline Conductas-Objetivo & Pretrat. & Postrat. & $\begin{array}{l}\text { Seg. } \\
1 \text { mes }\end{array}$ & $\begin{array}{l}\text { Seg. } \\
6 \text { meses }\end{array}$ & $\begin{array}{l}\text { Seg. } \\
12 \text { meses }\end{array}$ \\
\hline Grado de Dificultad (rango 0-10) & 41 & 8 & 11 & 8 & 2 \\
\hline 1. Hacerme una analítica & 10 & 0 & 3 & 3 & 1 \\
\hline 2. Ponerme una inyección & 9 & 0 & $\mathbf{0}$ & 1 & 0 \\
\hline $\begin{array}{l}\text { 3. Permanecer en una situación de limpieza } \\
\text { de heridas o cortes }\end{array}$ & 8 & 5 & 5 & 3 & 1 \\
\hline 4. Entrar en un hospital para ser atendido & 8 & 3 & 3 & 1 & 0 \\
\hline $\begin{array}{l}\text { 5. Ver en TV o en alguna revista } \\
\text { noticias relacionadas con la sangre }\end{array}$ & 6 & 0 & 0 & 0 & 0 \\
\hline $\begin{array}{l}\text { Frecuencia de Evitación (rango 0-25) } \\
(0=\text { nunca, } 5=\text { siempre) }\end{array}$ & 24 & 5 & 2 & 4 & 0 \\
\hline $\begin{array}{l}\text { Frecuencia de Episodios de Desmayo (rango } 0 \\
(0=\text { nunca, } 5=\text { siempre) }\end{array}$ & 20 & 3 & 0 & 3 & 1 \\
\hline
\end{tabular}


paciente se situaron en los valores medios de los 16 factores. Tan sólo en el factor A (sizotimia- afectotimia) y en el B (capacidad mental) nuestro paciente se destacaba ligeramente del grupo. En el factor A puntuó más bajo acercándose más al polo de la sizotimia, lo que está en consonancia con su carácter reservado. En cuanto al factor B puntuó más alto que la media del grupo, apuntándonos una alta capacidad de comprensión y aprendizaje de las ideas que se demostró por la rápida asimilación y comprensión del mecanismo del tratamiento una vez explicado en la primera sesión.

Finalmente y a modo orientativo, se formularon algunas cuestiones de interés clínico:

Percepción de autocontrol

( 0 = nada, 5 = muchísimo)

Nivel de interferencia del problema 3 en su vida ( $0=$ nada, $5=$ muchísimo $)$

Tendencia al desmayo

( 0 = nunca, 5 = siempre)

evitación

Estrategia actual de afrontamiento
Nivel de compromiso con

el tratamiento

( 0 = nada, 5 = totalmente)

Nivel de motivación

( 0 = nada, $5=$ muy motivado)

\section{Hipótesis Explicativa}

Terminada la fase de evaluación se llevó a cabo una explicación detallada y clara sobre el problema y sobre los diversos factores que lo mantienen así como sobre conceptos tales como la diferencia entre la ansiedad normal y la patológica, la fobia y más específicamente la hematofobia.

En este paciente se da el inicio del problema en la infancia, si bien no está claro el origen de la fobia, aunque pudiera deberse a una conducta aprendida de la forma de actuar de la madre y el hermano mayor ante las situaciones relacionadas con la sangre, ya que ambos padecen el mismo problema.

Desde los primeros episodios hasta el momento antes del tratamiento, el miedo ha persistido en el tiempo y su situación

Tabla 4. Puntuaciones obtenidas en el cuestionario $16 \mathrm{PF}$

\begin{tabular}{llc}
\hline \multicolumn{1}{c}{ Factores de Personalidad } & $\begin{array}{c}\text { Puntuación directa de } \\
\text { la media del grupo }\end{array}$ & $\begin{array}{c}\text { Puntuación directa } \\
\text { del paciente }\end{array}$ \\
\hline A. Sizotimia - Afectotimia & 10,33 & 6 \\
B. Inteligencia & 9,77 & 11 \\
C. Fuerza del ego & 16 & 11 \\
E. Sumisión - Dominancia & 13,44 & 12 \\
F. Desurgencia - Surgencia & 14,22 & 11 \\
G. Fuerza del Superego & 14,55 & 14 \\
H. Trectia - Parmia & 13,44 & 12 \\
I. Harria - Premsia & 11,22 & 10 \\
L. Alaxia - Protensión & 11,33 & 10 \\
M. Praxernia - Autia & 12,66 & 10 \\
N. Sencillez - Astucia & 10,33 & 12 \\
O. Adecuación Imperturbable -Tendencia a la culpabilidad & 9,77 & 8 \\
Q1. Conservadurismo - Radicalismo & 10,44 & 9 \\
Q2. Adhesión al grupo - Autosuficiencia & 11,11 & 8 \\
Q3. Baja integración - Mucho control de su autoimagen & 11,88 & 13 \\
Q4. Tensión Energética & 11,22 & \\
\hline
\end{tabular}


ha empeorado. Esto puede ser debido a que su fobia no se ha mostrado demasiado incapacitante para el paciente al no tener ningún trastorno médico que requiera enfrentarse a menudo a las situaciones fóbicas, lo que unido a lo relativamente fácil que es evitar esas situaciones, ha contribuido al mantenimiento del problema.

El resultado de todo esto es que ante el simple hecho de que el paciente tenga que curarse una herida por pequeña que sea, genera una respuesta de ansiedad llegando incluso al desmayo, lo cual actúa como escape de la situación aversiva, quedando reforzada negativamente la ansiedad mediante la evitación de la exposición con el estímulo conflictivo, lo que contribuye aún más a mantener la fobia.

A esto habría que añadir la anticipación de las posibles consecuencias negativas futuras (por ejemplo: incapacidad de hacer frente a las situaciones y a los síntomas, el temor al desmayo) y el recuerdo de las situaciones anteriores en que se ha enfrentado a situaciones fóbi- cas, que hacen que el paciente hasta el momento del tratamiento se perciba con ausencia de control sobre su problema.

\section{Tratamiento}

Con este paciente se utilizó la técnica de «tensión aplicada», un tratamiento combinado de "exposición gradual en vivo» $\mathrm{y}$ «tensión muscular» (Öst y Sterner, 1987), con el doble objetivo de:

a) Controlar los síntomas de ansiedad y detectar las primeras señales del síndrome vasovagal para evitar la pérdida de conocimiento y el desmayo con la utilización de la tensión muscular, para lo cual se enseñó la técnica de «tensión muscular» (Öst, Sterner y Fellenius, 1989. Adaptada por Borda, Antequera y Blanco, 1994) (Tabla 5), y

b) Enfrentarse a diversas situaciones relacionadas con la sangre que hasta el inicio del tratamiento habían sido evitadas, para lo cual se utilizó la combinación de la técnica de «tensión muscular»

Tabla 5. Pasos de la Técnica de Tensión Muscular

(Öst, Sterner y Fellenius, 1979 adaptada por Borda, Antequera y Blanco, 1994)

\section{PASOS DE LA TÉCNICA DE TENSIÓN MUSCULAR}

1. Sentar al paciente en una silla o sillón.

2. Colocar con las manos abiertas sobre las piernas, cerrar el puño, tensar los músculos de los brazos, (por ejemplo: imaginar que se tiene una moneda en la mano y no quiere que se caiga). Permanecer durante 10-15 sgs. (hasta que sienta el aumento de calor en la cara).

3. Aflojàr los brazos y relajar la tensión de los músculos (pero sin llegar a estar relajado sino volver al estado normal). Permanecer durante 15-20 sgs.

4. Tensar los músculos de las piernas, levantar unos centímetros los pies del suelo y apretar las rodillas entre sí (por ejemplo: imaginar que se tiene una moneda entre las dos rodillas y no quiere que se le caiga). Permanecer durante 10-15 sgs.

5. Aflojar las piernas y relajar la tensión de los músculos. Permanecer durante 15-20 sgs.

6. Tensar el cuerpo, intentar levantarse del asiento, elevando las nalgas sin presionar los pies contra el suelo ni apoyarse en los brazos. Permanecer durante 15-20 sgs.

7. Aflojar el cuerpo y relajar la tensión de los músculos. Permanecer durante 15-20 sgs.

8. Repetir el ejercicio pero esta vez tensando a la vez los músculos de los brazos, las piernas y el cuerpo. Permanecer durante 10-15 sgs.

9. Aflojar todo el cuerpo y relajar la tensión de todos los músculos del cuerpo. 
junto con la «exposición gradual en vivo" (Echeburúa y Corral, 1993).

Hay que mencionar que se le entrenó también en la técnica respiración tranquilizadora (Foa y Wilson, 1992) como estrategia para controlar, durante la exposición, los síntomas de la primera fase de la respuesta hematofóbica. Sin embargo, no se empleó la relajación para disminuir la ansiedad puesto que se favorecería el paso a la segunda fase de las respuesta bifásica con la aparición de los síntomas más específicos de esta fase: sudor frío, palidez de la cara, etc., y por tanto, se estaría favoreciendo el desmayo.

\section{DIARIO DE SESIONES}

La intervención se llevó a cabo durante 6 semanas con 1 sesión por semana de 90 minutos aproximadamente de duración cada sesión. El paciente formó parte de un grupo de $3 / 4$ personas con un nivel de severidad de la hematofobia similar, nivel alto.

\section{$1^{a}$ Sesión}

En primer lugar se le dio información al paciente sobre varios conceptos:

(1) El concepto de ansiedad y la importancia de ésta como mecanismo de defensa y como medio para preparar al organismo para estar alerta en situaciones de riesgo, así como la diferencia entre ansiedad normal, anteriormente descrita, y ansiedad patológica, explicándole cómo esta última es una respuesta desmesurada y puede llegar a interferir en la vida del individuo provocando diversas alteraciones.

(2) La diferencia entre miedo y fobia, explicando cómo la respuesta de la fobia es irracional y desproporcionada al estímulo fóbico.
Posteriormente se le explicó detalladamente en qué consiste la fobia a la sangre, su naturaleza y sintomatología:

(3) La fobia a la sangre se asemeja al resto de las fobias en:

- Conductas de evitación y escape de las situaciones temidas.

- Pensamientos irracionales relacionados con el enfrentamiento a estímulos fóbicos ( «creo que me voy a volver loco", "no creo ser capaz de aguantarlo sin marearme»)

- Presencia de síntomas psicofisiologicos: sudoración, temblores, visión borrosa, etc.

Pero a diferencia de las otras fobias se produce un aumento de las tres medidas psicofisiológicas: tasa cardíaca, tasa respiratoria y presión sanguínea, que constituye la primera fase del patrón de respuesta específico que caracteriza a la hematofobia, una respuesta bifásica en cuya segunda fase se da un descenso rápido de los parámetros citados, especialmente de la tasa cardíaca o bradicardia (30-45 pulsaciones / minuto) y de la presión sanguínea que puede llevar al desmayo.

Como consecuencia de esto, la respuesta de evitación se produce más por el temor al desvanecimiento en sí que por el miedo a la sangre propiamente dicho.

Al paciente se le informó de su patrón específico de respuesta para que aprendiera a darse cuenta de los síntomas de ansiedad y pudiera controlarlos. También se le informó que, como en su caso, es un trastorno que aparece en la infancia y tiende a persistir en el tiempo debido a la estrategia de evitación ante los estímulos fóbicos, así como que es muy frecuente la existencia de antecedentes familiares, como en su caso serían la madre y el hermano mayor.

En cuanto al tratamiento, cuyo contenido detallado queda recogido en el Dia- 
rio de Sesiones (Tabla 6), se comenzó con el entrenamiento en "tensión aplicada», que consta de dos partes:

(1) aprender a tensar los músculos del cuerpo, y

(2) aprender a identificar los primeros síntomas de la caída de la presión sanguínea (del síndrome vasovagal) para aplicar la técnica de tensión en los momentos de exposición.

La exposición en vivo, es el núcleo del programa de tratamiento, ya que la exposición a las situaciones fóbicas es la respuesta incompatible con la evitación que está manteniendo el problema. Este enfrentamiento a las conductas-objetivo, al ser en intervalos de tiempo largos (3060 minutos) va a favorecer que el paciente se vaya sintiendo capaz de llegar a controlar sus síntomas de ansiedad, disminuyendo como consecuencia su miedo a las situaciones relacionadas con la sangre.

Se le aconsejó que utilizara ya desde esta primera sesión, la respiración tranquilizadora, para que lo usara como estrategia de afrontamiento complemen- taria ante los primeros síntomas de la activación fisiológica ya que favorece un mayor control voluntario de la frecuencia respiratoria y mayor aporte de oxígeno a las fibras musculares.

Tras la información detallada y el entrenamiento en las técnicas (respiración tranquilizadora y tensión muscular), se le explicó cómo aplicar estas técnicas en algunas situaciones fóbicas y ésto se completó con la exposición a una situación ansiógena para poner en práctica las mismas: visualizar un vídeo de aproximadamente $25 \mathrm{mn}$. de duración con escenas relacionadas con la sangre graduadas de menor a mayor ansiedad. Las secuencias del vídeo eran: En primer lugar, una visión general de un Hospital, seguido de un quirófano vacío pero con el instrumental preparado. Tras esto, se ven escenas de una jeringuilla en el envoltorio, de una extracción de sangre y, por último, de una operación, desde que preparan al paciente, hasta la operación de un cáncer de intestinos.

Conviene señalar en este sentido que las situaciones fóbicas a lo largo de todo el tratamiento se van a ir incorporando de menor a mayor grado de malestar, de manera que el paciente pueda ir superan-

Tabla 6. Diario de sesiones

\begin{tabular}{|c|c|c|}
\hline Sesión & Pasos & Contenido de las sesiones \\
\hline $1^{2}$ & $\begin{array}{l}\text { I } \\
\text { II }\end{array}$ & $\begin{array}{l}\text { Explicaciones sobre diversos conceptos } \\
\text { Explicación y entrenamiento de las técnicas: Respiración tranquilizadora y Tensión } \\
\text { aplicada } \\
\text { (tensión muscular + exposición en vivo) } \\
\text { Tareas para casa }\end{array}$ \\
\hline $2^{u}-5^{a}$ & $\begin{array}{l}\text { I } \\
\text { II } \\
\text { III } \\
\text { IV }\end{array}$ & $\begin{array}{l}\text { Comentario sobre las dificultades surgidas en las tareas intrasesiones } \\
\text { Repaso de los ejercicios de entrenamiento a utilizar durante la exposición } \\
\text { Exposición en vivo a las conductas-objetivo definidas: } \\
\text { Cumplimentación del Registro de exposición. } \\
\text { Preparación de las tareas intrasesiones }\end{array}$ \\
\hline $6^{4}$ & $\begin{array}{l}\text { I } \\
\text { II } \\
\text { III } \\
\text { IV } \\
\text { V }\end{array}$ & $\begin{array}{l}\text { Comentario sobre las dificultades surgidas en las tareas intrasesiones } \\
\text { Repaso de los ejercicios de entrenamiento a utilizar durante la exposición } \\
\text { Exposición en vivo a la conducta-objetivo definida: } \\
\text { Cumplimentación del Registro de exposición } \\
\text { Valoración global del tratamiento. Informe Final } \\
\text { Hoja de Consejos Útiles para hacer frente a las situaciones relacionadas con la sangre }\end{array}$ \\
\hline
\end{tabular}


do conductas cada vez más complejas para que al disminuir su miedo se sienta capaz de afrontar situaciones más difíciles.

Desde los primeros instantes de la visualización se le enseñaba a identificar los síntomas de ansiedad específicos de cada una de las dos fases para que aprendiera a controlarlos. A medida que avanzaba el vídeo, el paciente se encontraba inquieto, le sudaban las manos y respiraba con rapidez e incluso empezó a encontrarse un poco mareado, por lo que le recordamos que pusiera en práctica las técnicas que había aprendido. Se le propuso que durante la visión de la película recurriera a algunas estrategias puntuales de afrontamiento (cerrar momentáneamente los ojos, interrumpir durante unos segundos la grabación, etc.) pero si no se sintiera incapaz de continuar se procedería a detener la película definitivamente (aunque se le pidió que describiera exactamente cada uno de los síntomas físiológicos con el fin de ir discriminando las respuestas más específicas relacionadas con el síndrome vasovagal, y que refirie- ra el contenido de los pensamientos que tenía presente: "no puedo controlarme», "me estoy sintiendo cada vez peor», «me voy a caer», «estoy más flojo», etc.). Durante las diferentes escenas del vídeo se fue anotando el nivel de ansiedad máximo en cada una de las secuencias con el fin de determinar el grado de control de los síntomas a pesar del malestar generado por la visualización de las mismas (Tabla 7). Tras unos minutos de la proyección, comprobó que no se sentía capaz de seguir con la visualización, por lo que se detuvo la película. A continuación, se mantuvo unos minutos tranquilizándose, hasta que disminuyó el nivel de ansiedad.

Al terminar la sesión, se le aconsejó que pusiera en práctica las técnicas que habían sido aprendidas al menos tres veces al día hasta la próxima sesión, y que aprovechara las ocasiones que se le presentaran entre las sesiones para poner en práctica lo aprendido (por ejemplo: leer o ver en algún libro un reportaje con escenas de sangre.)

Tabla 7. Reacciones durante la visión del vídeo de contenido hematofóbico

\begin{tabular}{llc}
\hline Contenido del vídeo & Reacción del paciente & $\begin{array}{r}\text { Nivel de } \\
\text { ansiedad } \\
\text { máximo }\end{array}$ \\
\hline I. Visión general de un Hospital. & $\begin{array}{l}\text { Sudoración, taquicardia, molestias en el estómago, } \\
\text { mayor bombeo de sangre. Nerviosismo e inquietud. } \\
\text { Pensamientos anticipatorios. }\end{array}$ & $5-6$ \\
& Se siente capaz de controlar los síntomas & $8-9$ \\
II. Visión general de un & Sudor frío, palidez facial, flojera en el cuerpo, mareo. & 8 \\
$\begin{array}{l}\text { III. Imágenes de una jeringuilla } \\
\text { tirada en una papelera. }\end{array}$ & $\begin{array}{l}\text { Se siente capaz de controlar los síntomas } \\
\text { Intenta seguir la proyección }\end{array}$ & 9 \\
& Se siente incapaz de controlar el malestar y continuar. & \\
IV. Imágenes de una extracción & Se detiene la película. \\
de sangre. & \\
V. Imágenes de una operación. &
\end{tabular}




\section{$2^{a}$ Sesión}

Se comenzó consultando con el paciente las dificultades surgidas en las tareas entre las sesiones. A este respecto se mostraba muy satisfecho porque había podido comprobar la efectividad de lo aprendido en la primera sesión en una situación real. Había presenciado cómo un amigo, mientras jugaba al fútbol, se había partido el labio sangrando mucho y él había podido presenciar la escena sin llegar a desmayarse, por lo que venía muy animado a continuar con el tratamiento. A continuación se hizo un breve repaso de las estrategias aprendidas para hacer frente a las situaciones hematofóbicas.

Tras estos se llevó a cabo la exposición a la $2^{\mathrm{a}}$ conducta-objetivo: Manipular el material de extracción. Hay que señalar, que aunque estas no eran exactamente las conductas-objetivo propuestas por el paciente, eran las acordadas por el grupo del que formaba parte. Durante aprox. $30 \mathrm{mn}$. estuvieron tocando jeringuillas, algodón, gomas, etc. Durante todo el tiempo se cumplimentó el Registro de exposición (Tabla 8).

Como estrategias complementarias se utilizaron el modelado del terapeuta o de otro compañero, y el empleo de autoinstrucciones positivas («esto no está resultando tan mal» «si ahora me esfuerzo, poco a poco lo voy a conseguir», «es cierto que siento malestar pero puedo controlarlo").

Al final de la sesión se prepararon tareas similares para practicar entre sesiones.

\section{$3^{a}$ Sesion}

Se comenzó comentando las dificultades surgidas al realizar las tareas intrase- siones y la percepción subjetiva de si hay mejoría para continuar con el repaso de las estrategias aprendidas. Resaltar aquí que, por primera vez, se lleva a cabo la sesión en un ambiente hospitalario, lo que constituía una posible dificultad añadida, ya que nuestro paciente también temía el entrar en un hospital.

En esta sesión, se comenzó la exposición, primero brevemente a la $2^{\mathrm{a}}$ conducta-objetivo, para luego pasar a una nueva: Simulacro de extracción de sangre, en la cual se le situaba en la situación de una extracción de sangre (colocación de la goma en el brazo, se le frota con un algodón, se le acerca la aguja al brazo, etc.). Si se mostraba demasiado ansioso inicialmente, se efectuaba el simulacro con otro del grupo o con un coterapeuta mientras él lo veía.

Durante todo el tiempo se fue cumplimentando el Registro de exposición (Tabla 8). Se concluyó la sesión planificando las tareas para casa de esa semana, estableciéndose que practicara en casa al menos dos veces al día, el simulacro realizado durante la sesión en grupo, manipular una jeringuilla, acercarse la aguja al brazo, pasar el dedo por las venas, etc. En todo momento debía estar atento a los síntomas para poner en práctica lo aprendido en la sesión.

\section{$4^{a}$ Sesión}

$\mathrm{Al}$ igual que en las anteriores, se comenzó con los comentarios sobre las dificultades surgidas en la realización de las tareas intrasesiones. Refería que al pensar en tener que hacer la tarea se ponía nervioso, pero que al realizarla gradualmente, se ha ido sintiendo más con-

Tabla 8. Nivel de ansiedad máximo durante las sesiones de exposición en el tratamiento

\begin{tabular}{cccccc}
\hline $1^{2}$ Sesión & $2^{\text {a }}$ Sesión & $3^{2}$ Sesión & $4^{4}$ Sesión & $5^{\text {a }}$ Sesión & $6^{\mathrm{a}}$ Sesión \\
\hline $8-9$ & $7-8$ & 9 & $7-8$ & $5-6$ & 6 \\
\hline
\end{tabular}


trolado. Tras ésto, se hizo un breve repaso de las estrategias aprendidas para hacer frente a las situaciones fobicas. Luego se procedió a la exposición. Se empezó por una breve exposición a la $3^{a}$ conducta-objetivo (en la que se registró un nivel inferior a 4 en el registro de ansiedad) para pasar a la $4^{\mathrm{a}}$ conducta: Extracción de sangre, para lo cual nos prestó su colaboración un ATS del Servicio del Hospital.

Durante esta exposición, mientras veía como a los otros miembros del grupo se les sacaba sangre, nuestro paciente entró en fase de riesgo de desmayo y él mismo pidió salir fuera de la sala donde nos encontrábamos, poniendo inmediatamente en práctica la técnica de «tensión muscular». Ya recuperado, practicó la «tensión aplicada» (tensión muscular durante la práctica de exposición). Pasado un tiempo se procedió a realizar la extracción.

Durante esta sesión se hizo una visita a la sección de Donantes de Sangre donde se le permitió manipular las bolsas con sangre y observar el proceso realizado durante las donaciones de sangre.

En el transcurso de la exposición, se cumplimentó el registro de la práctica de exposición en el que se recogía el nivel de ansiedad, la presencia de síntomas y las cogniciones así como la estrategia de afrontamiento utilizada (Tabla 8). Al finalizar la sesión se prepararon las tareas intrasesiones. Se le pidió que siguiera manejando y manipulando la jeringuilla y que intentara controlarse mientras pensaba en poder sacarse sangre. Por otro lado se comprometió a no apartar la vista ni evitar las situaciones imprevistas relacionadas con la sangre para comprobar si se sentía capaz de controlarse.

\section{$5^{a}$ Sesión}

Comenzó la sesión con los comentarios sobre las tareas realizadas intrasesiones y con el repaso de las estrategias aprendidas y utilizadas. Se sentía bastante mejor. Había realizado las tareas y puesto en práctica las técnicas cuando se sentía más nervioso. Era capaz de darse cuenta de las sensaciones y de controlarlas.

Tras esta fase se lleva a cabo la exposición a las conductas-objetivos. Al igual que en la sesión anterior, con la ayuda del ATS se procedió a la extracción de sangre del paciente tras lo cual se pasa a la siguiente conducta-objetivo: Visita al sector de Urgencias del Hospital. Durante todo el tiempo se cumplimentó el registro de la práctica de exposición (Tabla 8). El final de la sesión consistió en la preparación de las tareas intrasesiones. En este momento, se le propuso que se expusiera el mayor número de ocasiones que le surgieran e intentara hacerles frente, para que en caso de que hubieran dificultades, se pudieran comentar en la última sesión del tratamiento.

\section{$6^{a}$ Sesion}

Se comenzó como las anteriores, comentando las dificultades surgidas en las tareas y con un breve repaso de las estrategias utilizadas. El balance general de la semana fue bastante satisfactorio. A continuación, se procedió a la exposición a la conductaobjetivo de Extracción de Sangre para luego pasar a la última conducta-objetivo: Visitar la planta de Traumatología y presenciar la realización de curas de heridas.

Durante la sesión se cumplimentó el registro de exposición en el que se observaba que, aunque el nivel de dificultad de esta tarea era superior, se sentía más controlado ante las situaciones (Tabla 8).

Tras esto, llevamos a cabo la valoración global del tratamiento y el paciente rellenó el Informe Final (Borda, Antequera y Blanco Picabia, 1994), el cual recoge información acerca de la valoración del sujeto sobre diferentes aspectos generales 
y específicos (número de sesiones, grado de dificultad, etc.) que componen el tratamiento. Está diseñado en dos formatos similares, uno para su aplicación tras el tratamiento y otro para su aplicación tras el seguimiento de los 12 meses. En esta ocasión, se incorporan algunas cuestiones referentes al mantenimiento de los logros durante el período de seguimiento. También se le hizo entrega de una hoja con consejos útiles para hacer frente a las situaciones relacionadas con la sangre.

\section{RESULTADOS}

Los resultados de este estudio muestran la evolución del paciente desde la toma de contacto hasta un año de la finalización del tratamiento, en lo que se especifican los efectos a corto, medio y largo plazo (1, 6 y 12 meses).

A través de los resultados obtenidos en las diversas pruebas se observó una mejoría considerable en todas las medidas realizadas. Para ver la evolución del paciente recurrimos al criterio de valorar el porcentaje de mejoría del efecto de la intervención y del efecto global.

\section{a) Inventario de Fobia a la Sangre}

Se calculó este porcentaje de mejoría en el triple sistema de respuesta (respuestas psicofisiológicas, cognitivas y motoras). En este sentido, el porcentaje de mejoría del efecto del tratamiento fue del $89,25 \%$, $97,13 \%$ y $96,86 \%$, respectivamente. Por otro lado, el porcentaje del efecto global de la intervención a largo plazo muestra una mejoría del 98,16\%, 99,78\% y $99,10 \%$ respectivamente (Tabla 1 ).

\section{b) Cuestionario de Miedos}

Posteriormente, se llevó a cabo la determinación del porcentaje de mejoría en la subescala de hematofobia de Cuestionario de Miedos. El porcentaje de mejoría en el tratamiento fue del $75 \%$, que alcanzó el $100 \%$ en el efecto global de la intervención psicológica (Tabla 2).

\section{c) Escala de Conductas- Objetivo}

Finalmente se realizó el cálculo del porcentaje de mejoría en las tres subescalas de la Escala de Conductas-objetivo (Tabla 3). Subescala de Grado de Dificultad: El porcentaje de mejoría del efecto del tratamiento fue del $80,49 \%$. El porcentaje de mejoría global del 95,13\%. Subescala de Frecuencia de Evitación: El porcentaje de mejoría del efecto del tratamiento fue de un $79,17 \%$. El porcentaje de mejoría global de la intervención psicológica fue del $100 \%$. Subescala de Frecuencia de Episodios de Desmayo: El porcentaje de mejoría del efecto del tratamiento fue del $85 \%$, siendo el porcentaje de mejoría global del $95 \%$.

\section{DISCUSIÓN Y CONCLUSIONES}

Si revisamos la mayoría de los estudios llevados a cabo en los últimos años en el tratamiento de todo tipo de fobias, observamos que las técnicas más utilizadas son las de exposición por considerarlas como la estrategia más eficaz para eliminar la conducta de evitación (Borda, Báez y Echeburúa, 1993).

El programa de tratamiento elaborado para el grupo terapéutico del que formaba parte el paciente, clasificado como un nivel severo en hematofobia a partir de los datos del Inventario de fobia a la sangre, se mostró eficaz para nuestro paciente produciendo un cambio positivo en los principales síntomas que presentaba antes de la intervención. El alto grado de motivación y las expectativas positivas, que él tenía acerca del tratamiento, uni- 
das al importante apoyo de la familia, contribuyeron en gran medida al éxito del programa terapéutico.

La utilización de la técnica de exposición en vivo se muestra eficaz para eliminar la respuestas de evitación de las situaciones hematofóbicas (Borda, Antequera y Blanco Picabia, 1993). La mejoría significativa que se observa tras el tratamiento se sigue aumentando en el seguimiento, durante el cual el paciente siguió exponiéndose a diferentes tipos de situaciones relacionadas con la fobia a la sangre. El uso de la técnica de «tensión aplicada», que enseña a controlar las primeras señales de activación psicofisiológica ante la exposición a las situaciones temidas, contribuyen a la prevención del síndrome vasovagal, en un principio tan frecuente en el paciente. El ser capaz de controlar voluntariamente este tipo de síntomas refuerza el enfrentamiento con las situaciones problema.

Aunque el tratamiento está enfocado al control y eliminación de los síntomas psicofisiológicos y conductuales, podemos decir que existe una sincronía en el triple sistema de respuesta, ya que a la vez que mejoran las respuestas antes mencionadas, indirectamente mejoran también de forma muy significativa las respuestas cognitivas. Esto podría haberse visto favorecido por la rápida asimilación y comprensión del fundamento y funcionamiento de las técnicas que demostró el paciente desde el principio del tratamiento y que ya se apuntó en los resultados del inventario de personalidad.

Desde el momento en que el paciente va comprendiendo que es capaz de enfrentarse a las situaciones temidas, van disminuyendo las respuestas cognitivas de anticipación, sobre todo del desmayo, por lo cual, la asociacion de respuestas que contribuía al mantenimiento del problema, "miedo al desmayo - evitación», queda desmontada. Desde que comienza el enfrentamiento de forma gradual a las situaciones hematofóbicas con la consecuente puesta en práctica de las estrategias adecuadas en cada una de las fases, van mejorando los síntomas, lo que prepara al paciente para exposiciones posteriores a conductas cada vez más complejas, con la consiguiente desaparición del miedo.

Por otro lado, es preciso señalar el proceso de generalización de conductas positivas que se origina tras la intervención, puesto de manifiesto en la realización de otras tareas diferentes a las tratadas, que antes producían también una respuesta de ansiedad en el paciente. Relacionado con este proceso de generalización, observamos también que en las otras subescalas del Inventario de fobia a la sangre, la subescala de ansiedad social y la de agorafobia, la baja puntuación que se obtenía en el pretratamiento ha desaparecido completamente en el seguimiento de los 12 meses. Pero no queda demasiado claro hasta qué punto dichas puntuaciones más que ser algo independientes de la hematofobia, no eran sino dependientes de ella, como por ejemplo el ítem de agorafobia más puntuado era la entrada en hospitales, que si bien recordamos, era una de las conductasobjetivo de nuestro paciente para la exposición en el tratamiento de la hematofobia. De cualquier modo, las puntuaciones en estas subescalas no eran clínicamente significativas, así como no se detectó ningún otro tipo de fobia, ni otra patología en nuestro paciente.

Por último consideramos interesante destacar la percepción subjetiva de mejoría que él mismo tenía, mostrándose muy satisfecho con los resultados obtenidos tras la intervención. La estrategia habitualmente utilizada ( quedarse quieto esperando a ver que pasaba") de paralización, favorecía la caída de los parámetros de la fase primera, con la aparición de los síntomas de la segunda fase. Al lle- 
gar un riego menor de oxígeno al cerebro, se estaba contribuyendo a la sensación de mareo, con sudor frío, e incluso, poder terminar en desmayo.

Tras la explicación de la naturaleza del problema, de los motivos por los que se estaba manteniendo la sintomatología y de la puesta en marcha de las estrategias de afrontamiento ante la fobia, el paciente fue mejorando progresivamente. Los cambios significativos se alcanzaron ya durante el período de tratamiento, con un mantenimiento o consolidación de los logros durante la fase de seguimiento.

Por tanto, el manejo de las respuestas de ansiedad, el control de los síntomas previos al síndrome vasovagal a través de la tensión muscular y la exposición a las situaciones hematofóbicas contribuyen directamente al éxito terapéutico. Junto a esto, la percepción de control de los síntomas y las situaciones, las expectativas de autoeficacia y la confianza en sí mismo consolidan los resultados obtenidos en este paciente, en el que la mejoría fue significativa tanto para el afrontamiento y resolución de las situaciones evitadas como para el cambio en la interpretación anticipatoria de las posibles consecuencias que hasta entonces eran negativas.

\section{REFERENCIAS BIBLIOGRÁFICAS}

Agras, S., Sylvester, D. y Oliveau, D. (1969). Epidemiology of common fears and phobias. Comprehensive Psychiatry, 10, 151-156.

Borda, M., Báez, C. y Echeburúa, E. (1993). Tratamiento de exposición en un caso de fobia a la sangre. Análisis y Modificación de Conducta, 66, 583-607.

Borda, M., Antequera, R. y Blanco Picabia, A. (1994). La exposición gradual en vivo y la tensión aplicada en el tratamiento de la fobia a la sangre. Anales de Psiquiatria, 8 , 302-307.
Borda, M., Antequera, R. y Blanco Picabia, A. (1996). Orientaciones terapéuticas en el tratamiento de la hematofobia. Boletin de Psicología, 50, 67-86.

Borda, M., Rodríguez-Franco, L., Antequera, R. y Blanco Picabia, A. (1998, en prensa). Predicción de resultados: Gravedad de la hematofobia, variables de personalidad y éxito terapéutico. Análisis y Modificación de Conducta.

Echeburúa, E. y Corral, P. (1993). Técnicas de exposición en Psicología Clínica. En M.A. Vallejo y M.A. Ruiz (Eds.). Manual práctico de Modificación de Conducta (Vol. 2). Madrid. Fundación Universidad-Empresa.

Foa, E.B. y Wilson, R. (1992). Venza sus obsesiones. Barcelona: Robinbook.

Marks, I.M. (1987). Fears, phobias, and rituals: Panic, anxiety and their disorders. Oxford: Oxford University Press.

Marks, I.M. (1988). Blood-injury phobia: a Review. American Journal of Psychiatry, 145, 1207-1213.

Marks, I.M. y Mathews, A.M. (1979). A brief standard self-rating for phobic patients. Behaviour Research and Therapy, 27, 263267.

Öst, L.G., Sterner, V. y Lindahl, I.L. (1984). Physiological responses in blood phobics. Behaviour Research and Therapy, 22, 109-117.

Öst, L.G., Sterner, V. y Fellenius, J. (1989). Applied tension, applied relaxation, and tension-only in the treatment of blood phobia. Behaviour Research and Therapy, 27, 109-121.

Öst, L.G. (1992). Blood and injection phobia: Background and cognitive, physiological, and behavioural variables. Journal of Abnormal Pychology, 101, 68-74.

Page, A.C. (1994). Blood-injury phobia. Clinical Psychology Review, 54, 443-461.

Vázquez, M.I. y Buceta, J.M. (1990). Características peculiares de la fobia a la sangre, las enfermedades y las lesiones físicas: posibles implicaciones terapéuticas. $P$ siquis, 11, 42-47.

Weissman (1979). The Dysfunctional Attitude Scale: A validation study. Dissertation Abstract International, 40, 1389 B-1390 B. 